\title{
A RARE CASE OF PLUNGING RANULA WITHOUT ORAL COMPONENT- A CASE REPORT
}

Praveen Kumar ${ }^{1}$, Prashanth Veerabhadraiah², Nagaraj Therubheedhi Mayappa ${ }^{3}$, Saleem Saeed $A^{4}$, Vinay Kumar V5

${ }^{1}$ Associate Professor, Department ENT-HNS, Rajarajeswari Medical College, Bangalore. ${ }^{2}$ Assistant Professor, Department ENT-HNS, Rajarajeswari Medical College, Bangalore. 3 Professor and HOD, Department ENT-HNS, Rajarajeswari Medical College, Bangalore. ${ }^{4}$ Postgraduate Student, Department ENT-HNS, Rajarajeswari Medical College, Bangalore. 5 Postgraduate Student, Department ENT-HNS, Rajarajeswari Medical College, Bangalore.

HOW TO CITE THIS ARTICLE: Kumar P, Veerabhadraiah P, Mayappa NT, et al. A rare case of plunging ranula without oral component- A case report. J. Evolution Med. Dent. Sci. 2017;6(50):3875-3877, DOI: 10.14260/Jemds/2017/837

\section{PRESENTATION OF CASE}

A 15-year old female reported with a 3-year history of swelling in submental and bilateral submandibular region. The swelling was completely asymptomatic and there was a history of gradual increase in size of swelling. Patient does not give history of any surgical intervention before.

Patient was in good health and had no history of any systemic disorders. Family history and personal history were not remarkable.

On examination, general condition was good and vital signs were stable. On local examination, a diffuse swelling of $6 \times 5 \mathrm{~cm}$ in size was present in submental and bilateral submandibular region. Overlying skin was normal without local rise of temperature (Fig. 1). Swelling was brilliantly transilluminant (Fig. 2). Intra orally, no swelling was evident in sublingual area bilaterally. (Fig. 3). There was no carious tooth. Oral mucosa and gingiva was normal and salivary duct openings were patent with good oral hygiene.
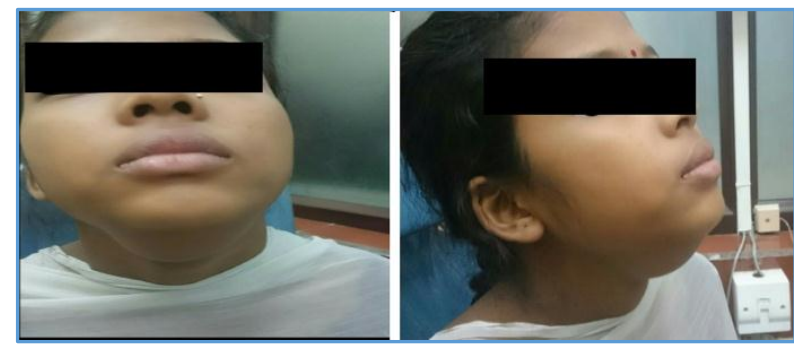

Figure 1. External Appearance of Swelling

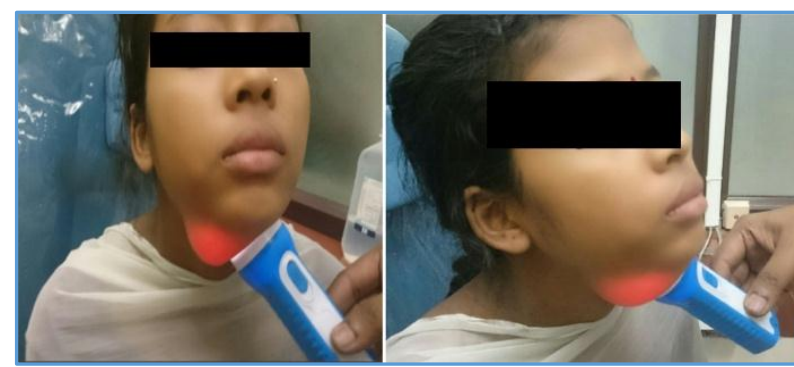

Figure 2. Brilliant Transilluminancy of Swelling

Financial or Other, Competing Interest: None.

Submission 12-05-2017, Peer Review 08-06-2017,

Acceptance 14-06-2017, Published 22-06-2017.

Corresponding Author:

Dr. Saleem Saeed A,

Room No. 501,

PG-Quarters,

Rajarajeswari Medical College Campus,

Kambipura, Mysore Road,

Bangalore-560074.

E-mail: salsai372@gmail.com

DOI: $10.14260 /$ jemds $/ 2017 / 737$

(c) (i) $(9)$

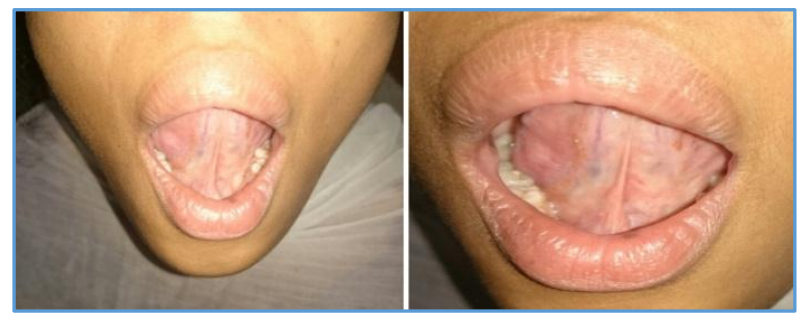

Figure 3. Intraoral Appearance (No Evidence of Swelling)

Ultrasonography of swelling showed multilobular cystic collection in bilateral submandibular region with thick internal echoes (Fig. 4) and the FNAC report showed numerous foamy macrophages in mucinous background without any inflammatory or epithelial cells. CECT of neck with plain and contrast enhanced serial sub-mm axial sections from orbital margin to thoracic inlet with $40 \mathrm{cc}$ of non-iodine contrast injection intravenously and $1 \mathrm{~mm}$ reconstructed sections showed large well-defined, multilobulated, thin-walled cystic lesion measuring $7.8 \times 3.5$ $\times 2.7 \mathrm{~cm}(\mathrm{TR} \times \mathrm{AP} \times \mathrm{CC})$ with multiple thin septae in subcutaneous plane of submental region extending to bilateral submandibular region. Cystic component showed minimal enhancement on post-contrast study. No evidence of calcification or haemorrhage was noted (Fig. 5).

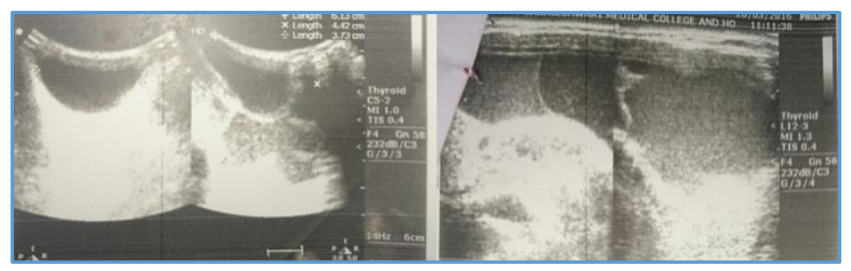

Figure 4. USG Neck showing Multilobular Cystic Collection in Bilateral Submandibular Region with Thick Internal Echoes
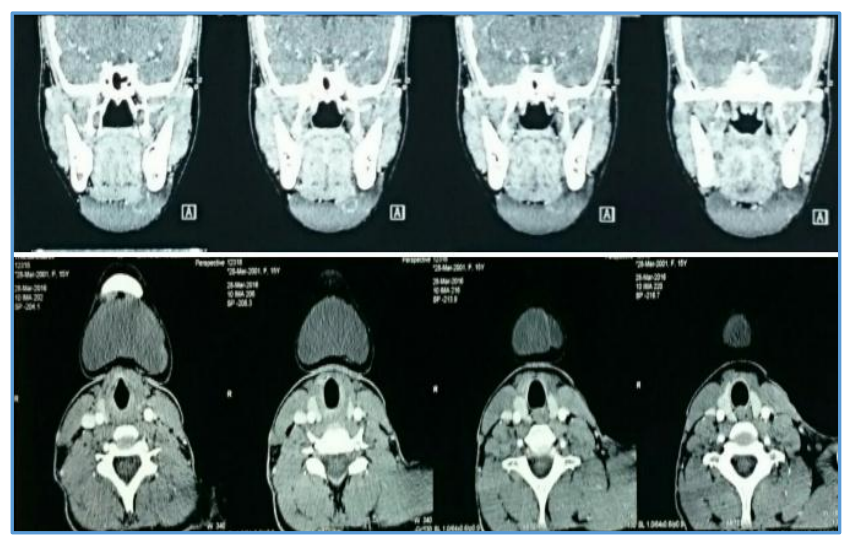


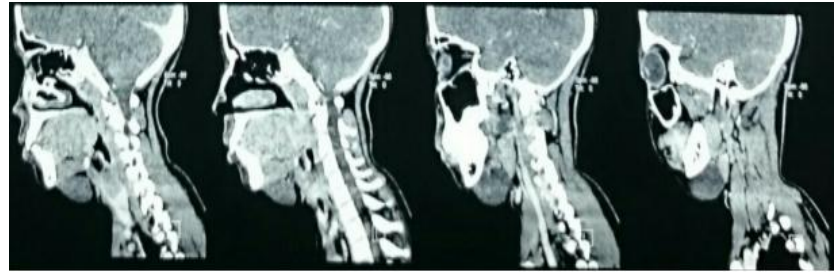

Figure 5. CECT Images showing Large Well-defined, Multilobulated, Thin-walled Cystic Lesion Measuring with Multiple Thin Septae in Subcutaneous Plane of Submental Region Extending to Bilateral Submandibular Region

\section{DIFFERENTIAL DIAGNOSIS}

Based on the clinicoradiological and CECT findings, a tentative clinical diagnosis of plunging ranula was made with strong differential diagnoses of lymphangioma, dermoid, epidermoid cyst, thyroglossal cyst and cystic hygroma. Other differential diagnoses of cervical ranula must include branchial cleft cyst, submandibular sialadenitis, intramuscular haemangioma, cystic or neoplastic thyroid disease, infectious cervical lymphadenopathy, haematoma, lipoma, and laryngocoele.[1]

\section{PATHOLOGICAL DISCUSSION}

A ranula by definition is a mucus filled cavity, a mucocoele, in the floor of the mouth in relation to the sublingual gland. The name "ranula" has been derived from the Latin word "rana" which means "frog." The swelling resembles a frog's translucent underbelly or air sacs. Ranulas are characteristically large $(>2 \mathrm{~cm})$ and appear as a tense, fluctuant and dome-shaped vesicle, sometimes with a blue hue.

Ranulas are cystic lesions of sublingual gland origin, simple ranulas are true retention cysts appearing as transparent thin-walled cysts, typically unilateral, within the floor of mouth. The cervical or plunging ranula is a mucus extravasation pseudocyst that arises as mucus escapes through a ruptured sublingual duct. Plunging ranulas may extend through the gap between the posterior edge of the mylohyoid muscle and anterior edge of the hyoglossus muscle in to the superior cervical neck. The exact prevalence of plunging ranula is not known, however, these lesions are considered uncommon.[2] Because most plunging ranulas either accompany a swelling in the floor of mouth or are associated with a history of treatment of intraoral ranula, it is not difficult to diagnose such a lesion. On the other hand, the plunging ranulas in which there is no clinical evidence of an oral connection, diagnosis becomes a challenge.[1]

Ranulas have a prevalence of about 0.2 cases per 1000 persons and accounts for $6 \%$ of all oral sialoceles.[2] It has been reported from 2 to 61 years of age with a slight female preponderance. ${ }^{[3]}$ The cervical variant tends to occur a little later in the third decade.

The aetiology is unknown, but it has been described in association with congenital anomalies, trauma, and disease of the sublingual gland.[1] The pathophysiology involved in extravasation is hypertension in the duct due to obstruction leading to acinar rupture in the salivary gland and then extravasation of the mucus.[1] The initial stage is a traumatic rupture of the excretory duct and the second stage is the extravasation and subsequent accumulation of saliva within the tissue.[1]

Plunging ranulas arise in the neck by one of the following four mechanisms. [2] Firstly, the sublingual gland may project through the mylohyoid, or an ectopic sublingual gland may exist on the cervical side of mylohyoid. This may explain the plunging ranulas that exist without an oral component. Secondly, a dehiscence or hiatus in the mylohyoid muscle may occur. This defect is observed along the lateral aspect of the anterior two-thirds of the muscle. Through this defect, the mucin from the sublingual gland may penetrate to the submandibular space. Thirdly, approximately $45 \%$ of plunging ranulas occur iatrogenically after surgery to remove oral ranulas or secondary to surgical procedures for sialolith removal, duct transposition and implant placement. Lastly, a duct from the sublingual gland may join the submandibular gland or its duct, allowing ranulas to form in continuity with the submandibular gland. Therefore, the ranula accesses the neck from behind the mylohyoid muscle.

The cervical ranula appears as an asymptomatic, continuously enlarging mass that may fluctuate in size. Most reported ranulas are $4-10 \mathrm{~cm}$ in size.[2] Similar to the oral ranula, the mass tends to cause a lateral swelling; however, it may cross the midline. The overlying skin is usually intact. The mass is fluctuant, freely movable, and nontender. In some instances, detecting salivary gland herniation of a portion of the sublingual gland through the mylohyoid muscle into the neck may be possible. Rarely, large-sized ranulas may cause dysphagia or airway obstruction.

In up to $45 \%$ of the cases, the patient's first presentation is an oral swelling. Plunging ranulas are associated with oral swelling in $34 \%$ of cases. Another $21 \%$ of the cases occur without any oral involvement. In the absence of oral swelling, the clinical diagnosis of ranula may be easily missed.[2]

Histopathologically, the cervical ranula appears identical to the mucus extravasation phenomenon. Biopsy of the lateral part of the neck may reveal only amorphous material with rare inflammatory cells and predominant histiocytes, which stains positive for mucin.[2] Biochemical analysis of fluid shows its high amylase and protein content.[1]

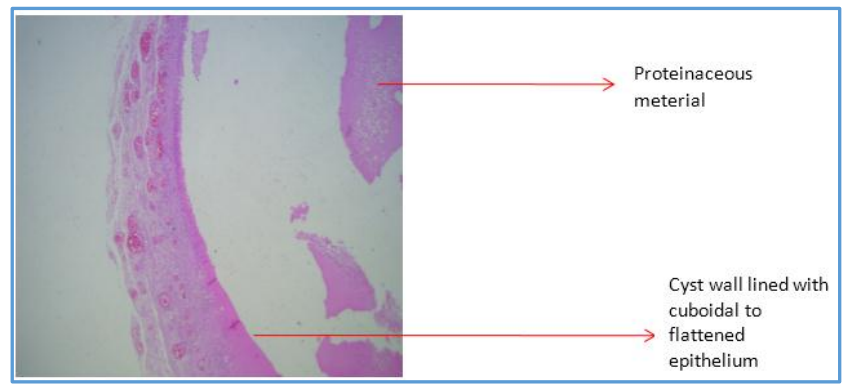

Figure 6. Histopathology Report

\section{DISCUSSION OF MANAGEMENT}

The excision of lesion was done via cervical approach under general anaesthesia, (Fig. 5 a \& b) and tissue was subjected for histopathological examination and its result came as mucocoele/ranula in submental region. (Fig. 6). 


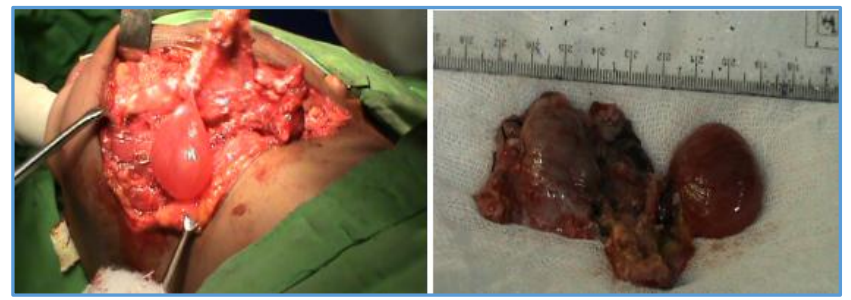

Figure 5a \& b. Excision via Cervical Approach and Excised Specimen.

Sialogram, ultrasonography, Magnetic resonance imaging (MRI), CT, and aspiration cytology can be helpful for diagnosis.[3] Takimoto suggested a simple radiographic technique for preoperative diagnosis of plunging ranula that involves injecting a contrast media in sublingual space.[2] A sialogram performed on a patient with a sialocyst reveals smooth displacement of the glandular ducts around the mass. No direct communication with the ductal system is demonstrated. However, the best method of demonstrating a communicating cyst is by sialography.[2] Ultrasonography is usually inconclusive to study sublingual glands due to their location. CT scan can help to understand the origin and extent of this cystic lesion. Although a plunging ranula may extend into the submandibular triangle and displace the submandibular gland, it does not intrinsically affect this gland. MRI is the most sensitive study to evaluate the sublingual gland and its states. ${ }^{[2]}$

Clinicians have been using several different methods for the treatment of cervical ranulas. These include excision of the ranula only, cryosurgery, marsupialisation with or without cauterisation of the lesion lining, excision of the oral portion of the ranula with the associated sublingual salivary gland or, rarely, the submandibular gland, intraoral excision of the sublingual gland and drainage of the lesion, and excision of the lesion via a cervical approach, sometimes combined with excision of the sublingual gland.[2]

Garofalo $\mathrm{S}$ et al in a study evaluated the effectiveness of orally administered Nickel Gluconate-Mercurius HeelPotentised Swine Organ Preparations D10/D30/D200 for treatment of ranula without surgery. The mechanism of action of which is to stimulate pseudocyst reabsorption and glandular repair, thus improving the physiologic functioning of the gland. They concluded that this is an effective treatment for ranula and oral mucocoeles.[4]

A study conducted by Fukase $\mathrm{S}$ et al showed a disappearance or marked reduction in ranula size with injection of OK-432. In their study, only half of the patients experienced local pain or fever which resolved within several days. Intracystic injection therapy with $0 \mathrm{~K}-432$ is relatively safe and can be used as a substitute for surgery in the treatment of ranula.[5]

The most common complications is the recurrence of the lesion $(5.78 \%)$ and sensory deficit of the tongue $(4.89 \%)$, followed by damage of Wharton's duct (1.82\%). Postoperative haematoma, infection, or dehiscence of the wound were seldom seen.[6] Excision of the ranula with the associated sublingual salivary gland is the most accepted method with low recurrence rate.[2] A biopsy of the cystic wall is recommended not only for histologic confirmation, but also to rule out presence of squamous cell carcinoma arising from the cyst wall and papillary cystadenocarcinoma of the sublingual gland, which may present as ranula.

Besides surgical management, $\mathrm{CO} 2$ laser has been used to vaporise ranulas.[3] In few cases, radiation therapy or intracystic injection of the streptococcal preparation, OK-432, has been used to treat this lesion.[6] The use of this sclerosing agent as a treatment approach for the cervical ranula is considered experimental. A recent study found orally administered Nickel Gluconate-Mercurius Heel-Potentised Swine Organ Preparations D10/D30/D200, a homotoxicological agent to be an effective treatment modality for ranulas. [7]

\section{FINAL DIAGNOSIS}

Based on the clinicoradiological, CECT findings and postoperative histopathology report, it was confirmed to be a case plunging ranula without its oral counterpart.

Though the cases of plunging ranula have been documented with moderate frequency, failure to differentiate the clinical features of oral and plunging ranulas may be a diagnostic pitfall. These lesions may be difficult to differentiate from benign and malignant salivary gland tumours, especially cystadenocarcinoma and mucoepidermoid carcinoma. A case of squamous cell carcinoma in the wall of ranula has also been reported. So, thorough radiological, biochemical, and histopathological investigations should be carried out for all cases of suspected plunging ranulas.

\section{REFERENCES}

[1] Sharma SK, Singhal G, Ragesh VG. A cervical ranula: a case report. International Journal of Medical Research \&Health Sciences 2016;5(7):109-13.

[2] Loney WW, Termini S, Sisto J. Plunging ranula formation as a complication of dental implant surgery: a case report. Journal of Oral and Maxillofacial Surgery 2006;64(8):1204-8.

[3] Sheikhi M, Jalalian F, Rashidipoor R, et al. Plunging ranula of the submandibular area. Dental Research Journal 2011;8(Suppl1):S114-8.

[4] Garofalo S, Briganti V, Cavallaro S, et al. Nickel gluconate-mercurius heel-potentised swine organ preparations: a new therapeutical approach for the primary treatment of pediatric ranula and intraoral mucocele. International Journal of Pediatric Otorhinolaryngology 2007;71(2):247-55.

[5] Fukase S, Ohta N, Inamura K, et al. Treatment of ranula wth intracystic injection of the streptococcal preparation OK-432. Ann Otol Rhinol Laryngol 2003;112(3):214-20.

[6] Zhao YF, Jia J, Jia Y. Complications associated with surgical management of ranulas. Journal of Oral and Maxillofacial Surgery 2005;63(1):51-4.

[7] Rho MH, Kim DW, Kwon JS, et al. OK-432 sclerotherapy of plunging ranula in 21 patients: it can be a substitute for surgery. American Journal of Neuroradiology 2006;27(5):1090-5. 